\title{
Human kallikrein 10 in surgically removed human pituitary adenomas
}

\author{
Fabio Rotondo, ${ }^{1}$ Antonio Di leva, ${ }^{3}$ Kalman Kovacs, ${ }^{1,2}$ Michael D. Cusimano, ${ }^{3}$ \\ Luis V. Syro, ${ }^{4}$ Eleftherios P. Diamandis, ${ }^{5}$ George M. Yousef ${ }^{1,2}$
}

${ }^{1}$ Department of Laboratory Medicine, and the Keenan Research Centre for Biomedical Science at the Li Ka Shing Knowledge Institute, St. Michael's Hospital, ${ }^{2}$ Department of Laboratory Medicine and Pathobiology, University of Toronto, ${ }^{3}$ Department of Neurosurgery, St. Michael's Hospital, University of Toronto, Toronto, Canada, ${ }^{4}$ Department of Neurosurgery, Hospital Pablo Tobon Uribe and Clinica Medellin, Medellin, Colombia, ${ }^{5}$ Departments of Pathology and Laboratory Medicine, Division of Clinical Biochemistry, Mount Sinai Hospital and Clinical Biochemistry, University Health Network, Toronto, Canada

\begin{abstract}
OBJECTIVE: Human kallikrein-like peptidase 10 (KLK10), a serine protease, plays an important role in the regulation of cell proliferation and tumor growth. In this work, we investigated KLK10 immunoexpression in various types of surgically removed human pituitary tumors. DESIGN: Specimens were fixed in formalin and embedded in paraffin. Immunostaining was performed by the streptavidin-biotin-peroxidase complex protocol using the LSAB + Kit and a KLK10-specific rabbit polyclonal antibody. RESULTS: Results showed that both treated and untreated prolactin-producing pituitary adenomas and carcinomas as well as TSH-producing pituitary adenomas and carcinomas were conclusively immunopositive for KLK10. Immunostaining was mainly localized in the cytoplasm and was clearly visible in many adenoma cells. In various other tumor types (oncocytoma, gonadotroph, somatotroph adenomas, and carcinomas), cytoplasmic immunopositivity was mild to moderate and seen only in a few unevenly distributed adenoma cells. Immunopositivity in the nuclei of various tumor types, as well as dual cytoplasmic and nuclear localization of KLK10 in some of the tumor types, was an intriguing finding. Immunoexpression in GH-producing adenomas exposed to octreotide, a long-acting somatostatin analog, was significantly increased when compared to unexposed GH-producing tumors. CONCLUSION: More studies are needed to ascertain the role of KLK10 in pituitary tumor development, prognosis, and progression. The question of whether genetic abnormalities and the microenvironment can affect KLK10 immunoexpression should also be investigated.
\end{abstract}

Key words: Human kallikrein 10, Immunohistochemistry, Pathology, Pituitary adenoma, Prognostic marker 


\section{INTRODUCTION}

The full characterization and mapping of the human tissue kallikrein gene family opened up new avenues for research. ${ }^{1,2}$ This gene family consists of 15 structurally homologous serine genes encoding for trypsin-like or chemotrypsin-like serine proteases and which are clustered together on the chromosomal locus 19q13.4. ${ }^{3,4}$ The designated nomenclature for these genes are KLK1-KLK15 and KLK1-KLK15 for their respective proteins. ${ }^{3}$ Kallikreins have been associated with a wide range of physiological and pathological processes. They were also shown to be associated with a number central nervous system disorders. ${ }^{5}$ They can function independently or in association with one or more proteolytic cascades. ${ }^{6}$ The KLK10 gene, originally named NES1 (normal epithelial cell-specific 1), encodes for human kallikrein-like peptidase 10 (KLK10), a serine protease, which can be found in various human tissues including breast, ovary, prostate, testis, glandular epithelium, choroid plexus, and cells within the central and peripheral nervous systems. ${ }^{7,8}$ Since its discovery, KLK10 has been found to play an important role in the regulation of cell proliferation and tumor progression. . $^{6-13}$ Although the physiological role of KLK10 is still unclear, it was reported to be associated with the control of normal cell growth and tumor suppression and to be downregulated during the progression of breast, prostate, and testicular cancers. ${ }^{10-12}$

Pituitary adenomas represent the most frequently occurring tumor type in the region of the sella turcica. They can arise from the different adenohypophysial cell types or their precursors and can produce various adenohypophysial hormones or may be endocrinologically silent, unassociated with hormone excess. The majority of pituitary tumors are expansively and slowly growing benign neoplasms which can cause endocrine and local symptoms. A minority of pituitary tumors grow more rapidly and invade adjacent structures. At present, it is difficult to prove by morphology which pituitary tumor is slowly or rapidly proliferating. Recently, several biomarkers have been investigated to assess pituitary tumor behavior.

In the present study, we investigated KLK10 immunoexpression in various surgically removed human pituitary tumor types to elucidate both its distribution and its level of expression and to provide evidence as to whether kallikreins are valuable biomarkers to assess prognosis.

\section{MATERIALS AND METHODS}

The study was based on a series of 96 surgically removed pituitary adenomas. Specimens included 14 prolactin (PRL)-producing adenomas not exposed to dopamine agonists, 10 growth hormone $(\mathrm{GH})$-producing adenomas not treated with octreotide, a long-acting somatostatin analog, 12 PRL-producing carcinomas, 10 oncocytic adenomas, 10 follicle-stimulating hormone (FSH) and luteinizing hormone ( $\mathrm{LH})$ producing adenomas, 12 thyrotroph hormone producing (TSH) adenomas, and $6 \mathrm{TSH}$-producing carcinomas. Also included in the study were 10 octreotide treated GH-producing adenomas and 12 dopamine agonist bromocriptine treated PRL-producing adenomas. ACTH secreting adenomas removed from patients with Cushing disease and silent subtype- 1 and silent subtype- 2 corticotroph adenomas were not included; they are part of a separate study.

\section{IMMUNOHISTOCHEMISTRY}

All specimens were fixed in $10 \%$ neutral buffered formalin, dehydrated in graded ethanol, and paraffinembedded. Sections of 4-6 $\mu \mathrm{m}$ thickness were stained with hematoxylin-eosin (H\&E), periodic acid-Schiff (PAS), and the Gordon-Sweet silver method for the demonstration of reticulin fibers. All specimens were immunostained using antisera directed toward $\mathrm{GH}$, PRL, ACTH, TSH, FSH, LH, the alpha chain of the glycoprotein hormones (alpha-subunit), Ki-67 using the MIB-1 antibody, and O(6)-methylguanine-DNA methyltransferase (MGMT), a DNA repair enzyme. Antibody sources and specifications were previously reported. ${ }^{14}$ In addition, all tumors were fixed in $2.5 \%$ glutaraldehyde, osmicated, routinely processed, and investigated by transmission electron microscopy. The aim of the immunohistochemical and ultrastructural investigation was to accurately classify the tumors and assess their apparent endocrine activity. Each tumor was classified according to the criteria defined by the World Health Organization (WHO) ${ }^{15}$

For the immunohistochemical demonstration of 
KLK10, the streptavidin-biotin-peroxidase complex protocol using the $\mathrm{LSAB}+\mathrm{Kit}$ (DAKO, Carpenteria, CA) was employed using an KLK10-specific rabbit polyclonal antibody (supplied by E.P Diamandis; dilution,1:150). Subsequent to antigen retrieval by microwaving in $0.1 \mathrm{mM}$ sodium citrate buffer $(\mathrm{pH}$ 6.0) as previously described, ${ }^{16}$ tissues were exposed to the primary antibody overnight at $4{ }^{\circ} \mathrm{C}$. Formalinfixed, paraffin-embedded normal human adult brain served as a positive control. Replacement of the primary antibody with PBS served as negative control. Further details of the immunohistochemical method were previously reported. ${ }^{14}$ The immunohistochemical findings were assessed independently by two of the authors (FR, KK). In accordance with the method described by Nakagawa et al, ${ }^{17}$ results of immunostaining for KLK10 were semi-quantitively expressed by a combined score resulting from multiplying the intensity of staining and frequency of reactive cells. Staining intensity was 4-tier, including 0 (negative), 1 (weak), 2 (moderate), and 3 (strong). The percentage of immunostained tumor cells was similarly ranked in a 4-tier including 1 (<10\%), 2 (10-50\%), 3 (51-90\%), and $4(>90 \%)$. The product of the previous scores served as a semi-quantitative assessment of staining. In keeping with the approach of Nakagawa et al, ${ }^{17}$ the scores for KLK10 staining fell into two groups, negative (0-1.9) and positive (2-12).

\section{STATISTICAL ANALYSIS}

Statistical analyses were performed with the use of the SPSS for Windows program (version 12.0; SPSS, Chicago, IL, USA).

This study analyzed associations between KLK10 immunopositivity in various pituitary adenomas. Also assessed were associations between age, gender, body weight, hormone profile, and MIB-1 labeling indices. The associations were analyzed by chi-square or Fisher exact tests, when appropriate.

\section{RESULTS}

Mild to moderate immunopositivity for KLK10 was localized in the cytoplasm and noted in all pituitary tumor types. Nuclear staining along with cytoplasmic staining was seen in various tumor types (oncocytic, gonadotroph, somatotroph adenomas). Nuclear immunostaining varied, but generally was present along with cytoplasmic staining. Intensity of nuclear staining correlated, in general, with that of cytoplasmic staining.

With the exception of the untreated GH-producing adenomas, moderate, heterogeneous immunopositivity was observed and its intensity varied, both from case to case and within the same tumor.

No statistically significant correlation was observed between KLK10 immunopositivity and clinical parameters including gender or age. Ki-67, a nuclear antigen expressed in the $\mathrm{G} 1, \mathrm{~S}$, and $\mathrm{M}$ phases of the cell cycle, is used as a marker for cell proliferation. Comparison between KLK10 immunoexpression and Ki-67 labeling index showed that KLK10 staining was noticeably higher in the cases that had higher Ki-67 labeling such as in carcinomas ( $\geq 10 \%)$, PRL-producing adenomas (Figure 1), and TSH-producing adenomas (Figure 2) (5-7\%) compared to the various other adenoma types (1-5\%). Immunopositivity was highest among the exposed and unexposed PRL-producing adenomas, and carcinomas, TSH-producing adenomas and carcinomas, and $\mathrm{GH}$ adenomas treated with octreotide preoperatively.

There was a clear difference in staining intensity between the GH adenomas exposed to octreotide

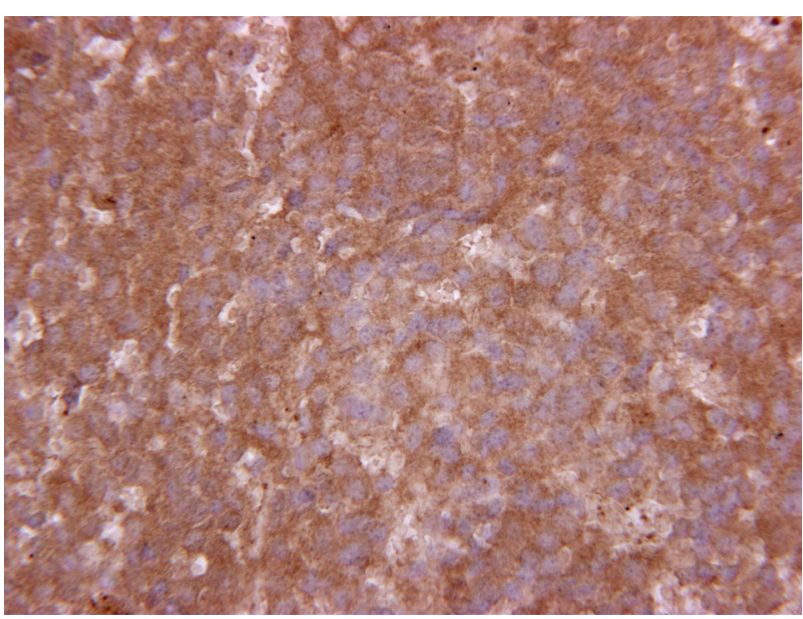

Figure 1. Untreated PRL-producing pituitary adenoma. The majority of cells are immunopositive for KLK10. Original magnification: $250 \mathrm{x}$. 


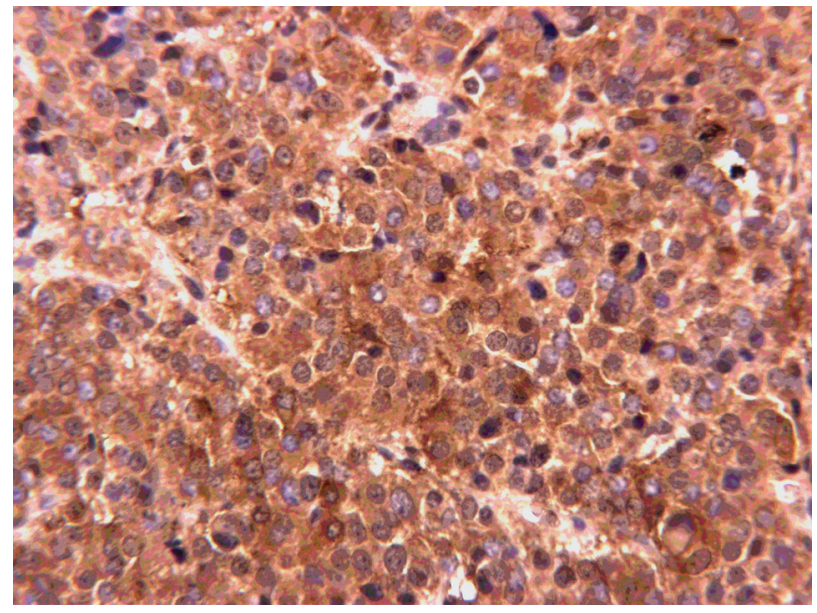

Figure 2. TSH-producing pituitary adenoma showing strong cytoplasmic KLK10 immunopositivity. Original magnification: 250x.

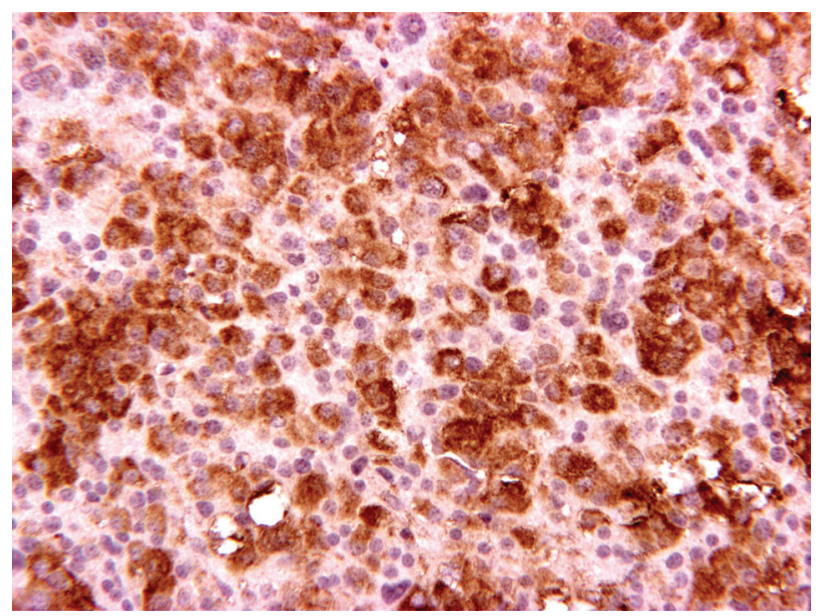

Figure 3. GH-producing pituitary adenoma treated with octreotide, a long-acting somatostatin analog showing strong cytoplasmic immunopositivity for KLK10. Original magnification: 250x.

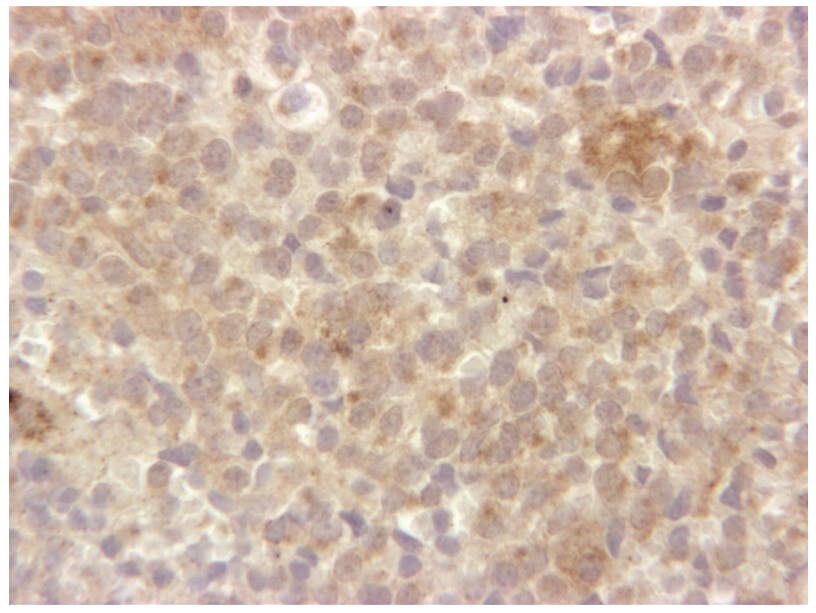

Figure 4. GH-producing pituitary adenoma not treated with octreotide showing very weak KLK10 cytoplasmic immunopositivity. Original magnification: 250x.

and the unexposed GH adenomas group (Figures 3, 4). Although based on the scoring method described above, untreated $\mathrm{GH}$ adenomas were immunonegative; nonetheless, the tumors did demonstrate weak immunopositivity in all cases. The negative control without the primary antibody for KLK10 staining did not show any positivity in the cytoplasm or nucleus of the tissues. The staining intensity and level of expression of KLK10 immunoreactivity in the various tumor types are summarized in Table 1.

\section{DISCUSSION}

KLK10 expression has been demonstrated in various tissues and tumor types, including breast, ovary, prostate, glandular epithelium, and cells within the central and peripheral nervous system. ${ }^{7,13}$ It has been

Table 1. Immunoexpression of Human Kallikrein 10 in pituitary adenomas

\begin{tabular}{lccccc}
\hline Adenoma Type & No. cases & M:F ratio & Median age & Age range & $\begin{array}{c}\text { KLK10 immunoexpression } \\
\text { (intensity } \times \text { frequency of stained cells) }\end{array}$ \\
\hline Untreated PRL & 14 & $6: 8$ & 40 & $25-54$ & 8.7 \\
Treated PRL & 12 & $6: 6$ & 37 & $25-54$ & 8.7 \\
PRL-secreting carcinoma & 12 & $6: 6$ & 47 & $25-69$ & 8.5 \\
TSH-secreting carcinoma & 6 & $6: 0$ & 55 & $49-61$ & 7.7 \\
TSH & 12 & $6: 6$ & 62 & $47-70$ & 6.3 \\
Treated GH & 10 & $4: 6$ & 59 & $40-77$ & 5.7 \\
Oncocytoma & 10 & $4: 6$ & 60 & $42-79$ & 4.0 \\
Gonadotroph & 10 & $4: 6$ & 58 & $40-78$ & 3.5 \\
Untreated GH & 10 & $4: 6$ & 50 & $29-85$ & 1.0 \\
\hline
\end{tabular}


shown to be downregulated in breast, cervical and prostate cancers and in acute lymphocytic leukemia, ${ }^{18}$ while upregulated in ovarian, colorectal, and gastric cancers. ${ }^{19}$ Earlier studies reported the presence of KLK10 in the nontumorous and adenomatous human pituitary. ${ }^{7,20,21}$ Petraki et $\mathrm{al}^{7}$ demonstrated strong immunopositivity in many cells of the human non-tumorous adenohypophysis. They concluded that the immunopositive cells were lactotrophs and corticotrophs and noted strong immunopositivity in epithelium-lined follicular and ductal formations of the pars intermedia which were also immunoreactive for PRL, while the pituicytes of the posterior lobe were immunonegative. Jones et $\mathrm{al}^{21}$ found that immunoreactive tissue kallikrein was localized in PRL-secreting adenomas of the human pituitary and suggested that localization was within the Golgi complex of those cells. In a study using female Wistar rats, Kizuki et al ${ }^{22}$ reported kallikrein immunopositivity in PRL-producing cells of the anterior pituitary. Hatala \& Powers ${ }^{23}$ found that in female rats both tissue kallikrein-like activity and PRL blood levels increased during puberty and the levels of tissue kallikrein-like activity changed similarly to those of PRL in both pituitary and blood during exposure to diethylstilbestrol, suggesting that kallikrein may be secreted by PRL-producing cells. Several studies showed that pituitary glandular kallikrein was induced by estrogen, suppressed by the dopaminergic neuroendocrine systems, and was elevated in estrogen induced PRL-producing lactotroph tumors. ${ }^{21-27}$

The present study demonstrated variable cytoplasmic KLK10 immunopositivity in a large number of surgically removed human pituitary tumors of varying types classified on the basis of clinical, immunohistochemical, and ultrastructural features. The results showed differences in both intensity of staining and frequency of immunopositive cells among the different tumor types. The cellular localization of KLK10 was not restricted to the cytoplasm of adenohypophysial cells and in several cases mild nuclear localization was also apparent. Dual nuclear and cytoplasmic expression demonstrated in some of the pituitary adenomas and carcinomas was an intriguing finding. This is a novel observation that has not been previously documented. This staining pattern was noted in the TSH adenomas and carcinomas as well as in the PRL carcinomas. The treated GH-producing adenomas had strong cytoplasmic immunostaining but which did however contain a few sparsely located cells that had the dual staining pattern. The observed nuclear staining of KLK10 is similar to what other researchers have documented for KLK4. Immunofluorescence and cell fractionation studies have shown that KLK4 localization was predominantly nuclear. ${ }^{28}$ KLK4 has been shown to play a role in tumor aggressiveness, chemotherapeutic drug resistance, metastasis, and promotion of cell proliferation. ${ }^{29}$ It is hypothesized that the nuclear localization of KLK10 may alter its function and contribute to tumor progression in a manner similar to that noted in KLK4. To further explain the possible cause of nuclear and/or cytoplasmic KLK10 staining present in several tumors, a comparison study using antibodies targeted against the $\mathrm{N}$-terminal, mid- and C-terminal regions of KLK10 protein should be undertaken. The findings are likely to determine whether conformational changes, truncated variants or full length antibodies may have an effect on staining patterns.

Immunoexpression of KLK10 was high in PRLproducing pituitary tumors. These findings are consistent with the results of Petraki et $\mathrm{al}^{7}$ and Jones et $\mathrm{al}^{21}$ who found a relationship between tissue kallikrein and PRL-secreting cells in both normal and adenomatous human pituitaries. Investigation of KLK10 immunoexpression in PRL-producing pituitary tumors exposed to dopamine agonists demonstrated no differences in immunoexpression between treated and untreated PRL-producing pituitary tumors and PRL-producing carcinomas. Overexpression of KLK10 protein was observed in all three groups with similar staining intensity and frequency. It is known that dopamine agonists inhibit secretion and PRL adenoma growth, decrease blood PRL levels, and cause tumors shrinkage. ${ }^{30}$ Our findings have shown that KLK10 immunoexpression is extremely strong in PRL-producing tumors and it was not possible to determine if there is any increase in immunoexpression in the PRL-producing tumors exposed to dopamine agonists.

Based on our analysis, it was noted that the more aggressive types of pituitary tumors had the highest KLK10 immunoexpression. Along with PRL-producing adenomas and carcinomas, TSH-producing 
adenomas and carcinomas were also highly immunoexpressive. Their staining pattern varied and contained both cytoplasmic and nuclear immunopositivity. TSH-producing pituitary tumors are often aggressive. They are rare and account for less than 1 percent of all pituitary tumors. ${ }^{31}$ Approximately 25 percent of adenomas secrete one or more pituitary hormones; about 15 percent secrete $\mathrm{GH}, 10$ percent secrete PRL, and others secrete gonadotropins. ${ }^{32}$ Cosecretion of TSH and PRL is about five times more common in women than in men. ${ }^{33}$ The presence of PRL in this tumor type may explain the intensity and pattern of KLK10 immunoexpression demonstrated in both adenomas and carcinomas. Decreased dopaminergic inhibition of PRL secretion due to increased estrogen levels are known to induce glandular kallikrein levels in the rat anterior pituitary. ${ }^{34}$ Estrogen hormones have an effect on both pituitary size and TSH blood levels and this may contribute to the high level of KLK10 present in TSH tumors. ${ }^{35}$

It was an intriguing finding that KLK10 immunoreactivity was elevated in $\mathrm{GH}$-producing adenomas exposed to octreotide compared to unexposed tumors. It is not known whether this increased immunoexpression is due to enhanced tumor suppressor activity. Although this possibility cannot be excluded, one has to take into account that the increased immunoreactivity is not apparent in dopamine agonist exposed PRL-producing tumors. It should also be noted that in pituitary adenomas and carcinomas with high cell proliferation rate, KLK10 immunoreactivity is high.

Our results showed that KLK10 immunoexpression was higher in $\mathrm{GH}$ producing pituitary adenomas exposed to octreotide, a long-acting somatostatin analog, compared to unexposed tumors. These results are consistent with the interpretation that in $\mathrm{GH}$ immunopositive adenomas, KLK10 plays a tumorsuppressing role. Several studies ${ }^{9,36}$ have indicated that increased kallikrein expression is a favorable prognostic indicator, even though there is evidence that increased kallikrein activity is associated with tumor progression and poor prognosis. It could be that KLKs may have a dual role possessing both stimulatory and inhibitory effects that modulate tumor cells in an autocrine and/or paracrine fashion in different tissues during tumor progression. ${ }^{37-42}$
In a recent study yet to be published, Di Meo et al found that KLK10 was elevated in ACTH-secreting tumors versus normal pituitaries. They also noted that KLK10 immunoreactivity was significantly lower in silent corticotroph adenomas. These novel findings suggest that in at least one pituitary tumor group, KLK10 immunoexpression may be used as a prognostic biomarker to distinguish between nonfunctioning corticotroph adenomas and functioning corticotroph adenomas.

More studies are needed to ascertain the role of KLK10 in pituitary tumor development and progression. The dichotomy of KLK10 expression pattern in relation to the various pituitary adenomas and carcinomas presents a challenge for researchers to examine their potential as biomarkers in these tumor types. The question of whether genetic abnormalities and microenvironment can affect KLK10 immunoexpression should also be investigated.

\section{ACKNOWLEDGEMENT}

The authors are grateful to the Jarislowsky and Lloyd Carr-Harris Foundations for their generous support. This work was also supported by grants from the Canadian Institute of Health Research, Kidney Foundation of Canada, the Kidney Cancer Research Network of Canada, and Prostate Cancer Canada.

\section{REFERENCES}

1. Yousef GM, Diamandis EP, 2001 The new human tissue kallikrein gene family: structure, function and association to diasease. Endocr Rev 22: 184-204.

2. Yousef GM, Chang A, Scorilas A, Diamandis EP, 2000 Genomic organization of the human kallikrein gene family of chromosome 19q13.3-13.4. Biochem Biophys Res Commun 276: 125-133.

3. Diamandis EP, Yousef GM, Clements J, et al, 2000 New nomenclature for the human tissue kallikrein family. Clin Chem 46: 1855-1858.

4. Yousef GM, Luo LY, Diamandis EP, 1999 Identification of novel human kallikrein-like genes on chromosome 19q13.3-q13.4. Anticancer Res 19: 2843-2852.

5. Yousef GM, Kishi T, Diamandis EP, 2003 Role of kallikrein enzymes in the central nervous system. Clin Chim Acta 329:1-8.

6. Borgoño CA, Diamandis EP, 2004 The emerging roles of human tissue kallikrein in cancer. Nat Rev Cancer 4: 876-890.

7. Petraki CD, Karavana VN, Luo LY, Diamandis EP, 
2002 Human kallikrein 10 expression in normal tissues by immunohistochemistry. J Histochem Cytochem 50: 1247-1261.

8. Petraki CD, Gregorakis AK, Papanastasiou PA, et al, 2003 Immunohistochemical localization of human kallikreins 6, 10 and13 in beign and malignant prostatic tissue. Prostate Cancer Prostatic Dis 6: 223-227.

9. Borgoño CA, Michael IP, Diamandis EP, 2004 Human tissue kallikreins: physiologic roles and applications in cancer. Mol Cancer Res 2: 257-280.

10. Goyal J, Smith KM, Cowan JM, Wazer DE, Lee SW, Band V, 1998 The role for NES1 serine protease as a novel tumor suppressor. Cancer Res 58: 4782-4786.

11. Dhar S, Bhargava R, Yunes M, et al, 2001 Analysis of normal epithelial cell specific-1 (NES1)/kallikrein 10 mRNA expression by in-situ hybridization, a novel marker for breast cancer. Clin Cancer Res 7: 3393-3398.

12. Luo LY, Meyts ER, Jung K, Diamandis EP, 2001 Expression of the normal epithelial cell-specific 1 (NES1, KLK10) candidate tumour suppressor gene in normal and malignant testicular tissue. Br J Cancer 85: 220-224.

13. Liu XL, Wazer DE, Watanabe K, Band V, 1996 Identification of a novel serine protease-like gene, the expression of which is down-regulated during breast cancer progression. Cancer Res 56: 3371-3379.

14. Kovacs K, Lloyd RV, Horvath E, et al, 1989 Silent somatotroph adenomas of the human pituitary: a morphologic study of three cases including immunocytochemistry, electron microscopy, in vitro examination and in situ hybridization. Am J Pathol 34: 345-353.

15. Kovacs K, Scheithauer BW, Horvath E, Lloyd RV, 1996 The World Health Organization classification of adenohypophysial neoplasms. A proposed five-tier scheme. Cancer 78: 502-510.

16. Rotondo F, Scheithauer BW, Kovacs K, Bell DC, 2009 Rab 3B immunoexpression in human pituitary adenomas. Appl Immunohistochem Mol Morphol 17: 185-188.

17. Nakagawa SA, Lopes A, Lopes de Carvalho A, et al, 2010 Nitric oxide synthases, cyclogenases-2, nitrotyrosine and angiogenesis in chondrosarcoma and their relation to prognosis. J Bone Joint Surg Am 92: 1738-1746.

18. Bharaz BB, Luo LY, Jung K, Stephan C, Diamandis EP, 2002 identification of single nucleotide polymorphisms in human kallikrein 10 (KLK 10) gene and their association with prostate, breast, testicular, ovarian cancers. Prostate 51: 35-41.

19. Feng B, Xu WB, Zheng MH, et al, 2006 Clinical significance of human kallikrein 10 gene expression in colorectal cancer and gastric cancer. J Gastroenterol Hepatol 21:1596-1603.

20. Luo LY, Grass L, Howarth DJC, Thibault P, Ong H, Diamandis EP, 2001 Immunofluorometric assay of human kallikrein 10 and its identification in biological fluids and tissues. Clin Chem 47: 237-246.

21. Jones TH, Figueroa CD, Smith CM, Cullen DR, Bhoola $\mathrm{KD}, 1992$ Tissue kallikrein is associated with prolactin- secreting cells within human growth hormone-secreting adenomas. J Endocrinol 134: 149-154.

22. Kizuki K, Kitagawa A, Takahashi M, Moriya H, Kudo M, Noguchi T,1990 Immunohistochemical localization of kallikrein within the prolactin-producing cells of the rat anterior pituitary gland. J Endocrinol 127: 317-323.

23. Hatala MA, Powers CA, 1988 Glandular kallikrein in estrogen-induced pituitary tumors: time course of induction and correlation with prolactin. Cancer Res 48: 4158-4162.

24. Clements JA, Fuller FJ, McNally M, Nikolanidis I, Funder JW, 1986 Estrogen regulation of kallikrein gene expression in the rat anterior pituitary. Endocrinology 119: 268-273.

25. Pritchett DB, Roberts JL, 1987 Dopamine regulates expression of the glandular-type kallikrein gene at the transcriptional level in the pituitary. Proc Natl Acad Sci USA 84: 5545-5549.

26. Fuller PJ, Matheson BA, MacDonald RJ, Verity K, Clements JA, 1988 Kallikrein gene expression in estrogeninduced pituitary tumors. Moll Cell Endocrinol 60: 225-232.

27. Powers CA, 1986 Anterior pituitary glandular kallikrein: trypsin activation and estrogen regulation. Mol Cell Endocrinol 46: 163-174.

28. Xi Z, Klokk TI, Korkmaz K, et al, 2004 Kallikrein 4 is a predominantly nuclear protein and is overexpressed in prostate cancer. Cancer Res 64: 2365-2370.

29. Zhang SQ, Cai B, Liu L, He YY, Yang YX, Wan XP, 2009 Kallikrein 4 overexpression in endometrial carcinoma and upregulation by estrogen via mitogen-activated protein kinase signal pathway. Int J Gynecol Cancer 19: 1377-1383.

30. Casanueva FF, Molitch ME, Schlechte JA, et al, 2006 Guidelines of the pituitary society for the diagnosis and management of prolactinomas. Clin Endocrinol 65: 265-273

31. Beck-Peccoz P, Brucker-Davis F, Persani L, Smallridge RC, Weintraub BD, 1996 Thyrotropin-secreting pituitary tumors. Endocr Rev 17: 610-638.

32. Sanno N, Teramoto A, Matsuno A, Inada K, Itoh J, Osamura RY, 1994 Clinical and immunohisto chemical studies on TSH-secreting pituitary adenoma: its multihormonality and expression of Pit-1. Mod Pathol 7: 893-899.

33. Adriannse R, Brabant G, Endert E, Bemelman FJ, Wiersinga WM, 1994 Pulsatile thyrotropin and prolactin secretion in a patient with a mixed thyrotropin-and prolactin-secreting pituitary adenoma. Eur J Endocrinol 130: 113-120.

34. Powers CA, Hatala MA, 1986 Dopainergic regulation of the estrogen-induced glandular kallikrein in the rat anterior pituitary. Neuroendocrinology 44: 462-469.

35. Abech DD, Moratelli HB, Leite SC, Oliveira MC, 2005 Effects of estrogen replacement therapy on pituitary size, prolactin and thyroid-stimulating hormone concentrations 
in menopausal women. Gynecol Endocrinol 21: 223-226.

36. Paliouras M, Diamandis EP, 2006 The kallikrein world: an update on the human tissue kallikreins. Biol Chem 387: 643-652.

37. Luo LY, Katsaros D, Scorilas A, et al, 2001 Prognostic value of human kallikrein 10 expression epithelial ovarian carcinoma. Clin Cancer Res 7: 2372-2379.

38. Roman-Gomez J, Jimenez-Velasco A, Agirre X, et al, 2004 The normal epithelial cell-specific 1 (NES1) gene, a candidate tumor suppressor gene on chromosome $19 q 13.3-4$, is downregulated by hypermethylation in acute lymphoblastic leukemia. Leukemia 18: 362-365.

39. Yousef GM, Stephan C, Scorilas A, et al, 2003 Differ- ential expression of the human kallikrein gene 14 (KLK $14)$ in normal and cancerous prostatic tissues. Prostate 56: 287-292.

40. Yousef GM, Scorilas A, Nakamura T, et al, 2003 The prognostic value of the human kallikrein gene 9 (KLK9) in breast cancer. Breast Cancer Res Treat 78: 149-158.

41. Yu H, Levesque MA, Clark GM, Diamandis EP, 1998 Prognostic value of prostate0specific antigen for women with breast cancer: a large United States cohort study. Clin Cancer Res 4: 1489-1497.

42. Yu H, Giai M, Diamandis EP, et al, 1995 Prostate-specific antigen is a new favorable prognostic indicator for women with breast cancer. Cancer Res 55: 2104-2110. 